Referencia para citar este artículo: Ospina-Ramírez, D. A., López-González, S., Burgos-Laitón, S. B., \& Madera-Ruiz, J.A. (2018). La paz entre lo urbano y lo rural: imaginarios de paz de niños y niñas sobre el posconflicto en Colombia. Revista Latinoamericana de Ciencias Sociales, Niñez y Juventud, 16(2), 943-960. doi:https://doi.org/10.11600/1692715x.16220

\title{
La paz entre lo urbano y lo rural: imaginarios de paz de niños y niñas sobre el posconflicto en Colombia*
}

\author{
DaVID ARTuro OSPINA-RAMíREZ ${ }^{* *}$ \\ Profesor- investigador Universidad Católica de Manizales, Colombia. \\ SERGIO LóPEZ-GONZALEZ ${ }^{* * *}$ \\ Estudiante Universidad de Caldas, Colombia. \\ SANDRA BIBIANA BURGOS-LAITÓN ${ }^{* * * *}$ \\ Profesora- investigadora Universidad Católica de Manizales, Colombia. \\ José AlEJANDro MADERA-RUIZ ${ }^{* * * * *}$ \\ Coordinador de proyectos Fundación Polifonía, Fundación Create-Cauca, Colombia.
}

\section{Artículo recibido en julio 4 de 2017; artículo aceptado en septiembre 22 de 2017 (Eds.)}

- Resumen (analítico): en este artículo visibilizamos algunos resultados de una investigación con la que buscamos conocer las concepciones que tienen los niños y las niñas de los departamentos de Caldas y Cauca, sobre la paz en un contexto de posconflicto en Colombia. Adoptamos una metodología cualitativa, con orientación hermenéutica comprensiva, mediante un estudio de caso y el análisis categorial de narrativas y productos gráficos y escritos de los sujetos participantes. Desarrollamos la investigación con 30 niños y niñas de la ciudad de Manizales y 30 niños y niñas de zonas rurales de Popayán, encontrando que los niños y niñas del campo piensan que los Acuerdos de Paz generarán desarrollo para sus comunidades y les garantizarán que los grupos armados no volverán a hacer daño; mientras que los niños y niñas en territorios urbanos expresan inicialmente cierta predisposición y miedo al pensar que puedan llegar a sus barrios personas desmovilizadas de

Este artículo de investigación científica y tecnológica (área de educación-sub área interdisciplinaria), presenta resultados de la investigación «La paz entre lo urbano y lo rural: imaginarios de paz de niños y niñas sobre el posconflicto en Colombia», de la Universidad Católica de Manizales, la Fundación Elefantes de Colores y el Colombo Americano Manizales, iniciada en febrero de 2016 y finalizada en el mes de mayo del año 2017.

* Magíster en Educación y Desarrollo Humano, Universidad de Manizales-Cinde. Magíster en Gestión de Proyectos, Universidad EAN y Université du Québec. Diseñador Industrial, Universidad Autónoma de Colombia. Docente investigador de la Especialización en Gerencia Educativa, Universidad Católica de Manizales. Líder de proyectos en Fundación Elefantes de Colores. Orcid: 0000-0002-1954-2489. Correo electrónico: david0206ospina@gmail.com

*** $\quad$ Estudiante de Antropología y Desarrollo Familiar de la universidad de Caldas. Integrante del semillero Paz y Desarrollo de la universidad de Caldas. Estudiante investigador del proyecto La paz entre lo urbano y lo rural: imaginarios de paz de niños sobre el posconflicto en Colombia. Orcid: 0000-0001-7512-2828. Correo electrónico: lopezgonzalezsergio91@gmail.com

**** Magíster en Educación de la Universidad Católica de Manizales. Especialista en Gerencia de Calidad de la Universidad Católica de Manizales. Licenciada en Biología y Química de la Universidad de Caldas, Coordinadora de calidad del Colegio Franciscano Agustín Gemelli de la cuidad de Manizales, docente de Administración Ambiental y de los Recursos Naturales de la Universidad Santo Tomas, Manizales. Actualmente, docente de la Especialización en Gerencia Educativa de la Universidad Católica de Manizales. Orcid: 0000-0002-2575-500X. Correo electrónico: danassbb@hotmail.com

****** Especialista en Gerencia Educativa de la Universidad Católica de Manizales. Ingeniero Físico de la Universidad del Cauca, coordinador académico sede El Bordo Paramédicos EMS, coordinador de proyectos en la Fundación Polifonía y Fundación Create-Cauca. Orcid: 0000-00019670-048X. Correo electrónico: amadera@unicauca.edu.co 


\section{DAVID ARTURo OSPINA-RAMÍREZ - SERGIO LÓPEZ-GONZÁLEZ - \\ SANDRa Bibiana BuRgos-LaITÓN - José ALEJANDRo MADERA-RUIZ}

los grupos armados; sin embargo, también muestran su disposición para acoger a estas personas y enseñarles nuevas maneras de construir paz.

Palabras clave: paz, violencia, infancia, creatividad (Tesauro de Ciencias Sociales de la Unesco).

\section{Peace between urban and rural settings: children's conceptions of peace for the post-conflict stage in Colombia}

- Abstract (analytical): This article highlights the results of a study that sought to identify the conceptions that the children of Caldas and Cauca have of peace in the post-conflict context in Colombia. The research used a qualitative methodology with comprehensive hermeneutical orientation based on a case study and the categorical analysis of narratives and graphic images and written texts produced the participants. The research was carried out with 30 children in the city of Manizales and 30 children in rural areas in Popayán. The study identified that rural children believe that the Peace Agreement will generate development for their communities and means that armed groups will not harm their communities again. Children in urban areas initially expressed certain attitudes and fear in thinking that demobilized persons from armed groups could live their communities, they also showed willingness to welcome these people and teach them new ways to build peace.

Key words: Peace, violence, childhood, creativity (Unesco Social Sciece Thesaurus).

\section{A Paz entre o urbano e o rural: concepções de paz entre crianças sobre pós-conflito na Colômbia.}

- Resumo (analítico): este artigo mostra alguns resultados de uma pesquisa concluída que buscou conhecer as concepções que meninos e meninas de Caldas e Cauca têm sobre a paz em um contexto pós-conflito na Colômbia. A pesquisa possui uma metodologia qualitativa com orientação hermenêutica abrangente, através de um estudo de caso e análise categórica de narrativas e produtos gráficos e escritos dos participantes. A pesquisa foi realizada com 30 crianças da cidade de Manizales e 30 crianças das áreas rurais de Popayán. Constatando que as crianças rurais acreditam que os Acordos de paz gerarão desenvolvimento para suas comunidades e permitirão ter certeza de que os grupos armados não prejudicarão novamente suas comunidades; enquanto meninos e meninas nas áreas urbanas inicialmente expressavam certa predisposição e medo ao pensar que pessoas desmobilizadas de grupos armados podiam chegar às suas comunidades, mas também mostraram vontade de receber essas pessoas e ensinar-lhes novas formas de construir a paz.

Palavras-chave: paz, violencia, infancia, criatividade (Thesaurus de Ciências Sociais da Unesco).

-1. Introducción. Conflicto armado en Colombia y su impacto en la niñez desde una mirada diferencial de etnia y territorio. El potencial creativo de la niñez para la construcción de paz. -2. Ruta metodológica. -3. Principales hallazgos y resultados. -4. Conclusiones y comentarios finales. -Lista de referencias.

«Y por sobre todo, observa con ojos brillantes a todo el mundo que te rodea, porque los secretos más grandes siempre están escondidos en los lugares menos probables. Aquellos que no creen en la magia nunca la encontrarán.»

Roald Dahl, Los Minpins

\section{Introducción}

La comprensión del fenómeno del conflicto armado y el momento histórico que atraviesa el país con la firma de los acuerdos de paz, supone retos importantes para los escenarios educativos, 
escenarios que, además de ser los espacios de construcción conjunta de una paz duradera instalada en la sociedad, son también responsables de la formación de las comunidades y de permitir que estas desplieguen sus capacidades. Es entonces fundamental generar estrategias, herramientas y metodologías adecuadas para dar a conocer las posturas de los niños y las niñas respecto a los acuerdos de paz y a sus maneras de aportar a la transformación de una cultura violenta permeada en el país. En el contexto colombiano, los actores de la guerra han jugado diferentes roles, y especialmente en la firma de los acuerdos de paz diferentes voces fueron escuchadas; sin embargo, los imaginarios que tiene la niñez sobre la construcción de paz en el país no han sido fuertemente visibilizados; por ende, la voz del sujeto adulto ha sido la principal postura de construcción de paz, y es por ello que investigar sobre las concepciones y las posturas que tienen los niños y niñas sobre los Acuerdos, permitirá también acercar sus voces a la construcción de paz y a la transformación que requiere el país.

El Departamento de Caldas y el Departamento del Cauca se han visto fuertemente afectados por la violencia; la Unidad para las Víctimas (2017) menciona que en el Departamento de Caldas se reporta un total de 134001 personas afectadas por el conflicto armado, mientras que en el departamento del Cauca se reporta un total de 296942 personas víctimas de la violencia; el mismo reporte permite encontrar que 1521563 son las personas entre los 0 y los 18 años de edad, afectadas por la guerra en el país (Unidad para las Víctimas, 2017). Lo anterior implica reconocer que Colombia se enfrenta a un reto enorme de formación, y especialmente de reconocimiento de las voces de niños y niñas sobre lo que implican los Acuerdos de Paz para sus territorios, así como la visibilización de sus posturas respecto a la construcción de paz en el posconflicto desde una mirada diferencial, entendiendo de antemano que la afectación de la guerra ha sido diferente para los contextos urbanos que para los contextos rurales.

\section{Conflicto armado en Colombia y su impacto en la niñez desde una mirada diferencial de etnia y territorio}

El país atraviesa un momento histórico que le lleva a enfrentarse a varios retos importantes para la construcción de una paz cercana a las comunidades, entre ellos el reconocimiento de más de 8532636 personas que se han visto afectadas por el conflicto armado (Unidad para las Víctimas, 2017).

En el escenario del posconflicto, el cese al fuego por parte de los grupos armados ha generado y seguirá generando un incremento en la cantidad de reportes de personas que se vieron afectadas por la violencia, ya sea en condiciones de desplazamiento o personas desmovilizadas de los grupos armados, y en general, personas con diversas formas de impacto por la guerra; la cifra actual de 1521623 niños, niñas y jóvenes menores de 18 años afectados por la guerra, indica que el 17,8\% de víctimas en el país son menores de edad (Unidad para las Víctimas, 2017). Las Instituciones Educativas se enfrentan a todos los cambios y retos que trae la firma de los acuerdos de paz, de manera directa desde la comprensión de su rol en la transformación de las vidas de los niños, las niñas y los jóvenes.

Las instituciones educativas, como centros de formación, se enfrentan a las particularidades de las huellas que el conflicto ha dejado en las personas; muchos de los niños y las niñas afectados por la violencia no cuentan con procesos de alfabetización, y solamente han conocido la guerra como manera de subsistencia; es el caso de los niños, niñas y jóvenes que hicieron parte de los grupos armados.

Existen diferentes variables que afectarán los procesos educativos de los niños, niñas y jóvenes, que permitirán llegar a una educación donde el respeto, la libertad y la tolerancia se hagan evidentes, desde una mirada crítica y transformadora de la educación, que les permita a los niños, niñas y jóvenes desplegar sus capacidades para construir paz y transformar el trayecto histórico de violencia que ha trazado el país. Es por esto que en desarrollo de la investigación buscamos conocer las concepciones y las posturas que tienen los niños y las niñas respecto al proceso que enfrenta el país, reconociendo la pertinencia de investigar a profundidad las maneras en que ellos y ellas interpretan la paz y el conflicto armado, y cuál es su postura respecto a la firma de los acuerdos de paz. 
Alvarado, Ospina, Quintero, Luna, Ospina-Alvarado y Patiño (2012), analizan el impacto del conflicto armado en las vidas de los menores:

En este panorama, los sectores populares están en medio de la guerra, del conflicto y la violencia generada por el sistema de injusticias, que está en la raíz del problema, pero que la otra violencia de las armas disfraza (...) Y en las esferas de lo invisible, encontramos a esos seres humanos, supuestamente la esperanza de la civilización, como carne de cañón en la vanguardia de los frentes de batalla. Reclutados indiscriminadamente, ingresan a los ejércitos regulares e irregulares porque no tienen alternativas económicas, sociales ni políticas. Son vulnerados sus derechos y obligados a cargar el fusil en vez de los útiles escolares. (p. 29)

El caso de las personas vinculadas al conflicto armado como miembros de los grupos militares no es distante del análisis planteado por los autores, siendo estas también víctimas del reclutamiento, de la vulneración de sus derechos. Incluso, el país se enfrenta a una variable importante a tener en cuenta, a la hora de considerar la totalidad de las víctimas de guerra:

Para responder a la pregunta de cuántos niños y niñas han sido reclutados por las Farc, el ELN y las Autodefensas Unidas de Colombia, revisamos las bases de datos de 10732 desmovilizados adultos pertenecientes al ELN, las Farc y las AUC, y comparamos la edad avalada por la Registraduría General del Estado Civil durante el acto de entrega con el tiempo de militancia al interior del grupo armado. Los resultados de ese ejercicio son concluyentes al señalar que: el 52,3\% de los combatientes adultos afiliados al ELN ingresó a las filas de este grupo armado siendo niño. El 50,14\% de los combatientes adultos en las Farc ingresó a las filas de este grupo armado siendo niño. El 38,12\% de los combatientes adultos en las Autodefensas Unidas de Colombia ingresó a las filas de este grupo armado siendo niño. Las AUC habrían reclutado menos niños y niñas debido a que contaban con un mejor acceso a una mano de obra adulta para la vinculación en cuanto disponían de los medios económicos para pagarla. (Springer, 2012, p. 28)

Por lo anterior, la mirada que la infancia tiene sobre los acuerdos de paz es importante y vital para la transformación de una cultura que se ha naturalizado desde la violencia. El reconocimiento de estas voces también permitirá identificar sus posibilidades para la transformación de las huellas del conflicto armado.

\section{Afectaciones del conflicto armado desde una mirada diferencial en la niñez}

La comprensión del impacto del conflicto armado permitirá encontrar nuevas maneras de educar para la paz, en un país que se enfrenta al posconflicto, un escenario desconocido y bastante amplio y complejo. Por ejemplo, no todas las regiones del país han tenido la misma afectación; al respecto, Springer (2012) manifiesta que:

En la Región Pacífica (Nariño, Cauca, Valle del Cauca y Chocó), el reclutamiento de niños y niñas indígenas sigue un patrón concluyente: el $29 \%$ de los niños y niñas reclutados son indígenas, una cifra nueve veces más alta que su peso en la composición étnica nacional (...) Parte de la explicación de la desproporcionada presencia de niños y niñas indígenas en grupos armados se encuentra en la tasa de rendimiento y la tasa de deserción: los niños y niñas indígenas son los que mejor resisten las difíciles condiciones del reclutamiento y los que menos desertan o abandonan las filas. Sus circunstancias socioeconómicas y culturales, así como la ubicación de sus territorios ancestrales, coinciden con la localización de corredores estratégicos y zonas de explotación de recursos. (Springer, 2012, p. 20)

Las condiciones de los territorios, las capacidades de las diferentes etnias para adaptarse a los cambios climáticos, a las fuertes jornadas de trabajo y a diversas condiciones de los grupos armados, exponen en mayor grado a algunas poblaciones étnicas más que a otras; en este punto, reconocemos la importancia de considerar las formas en que el conflicto ha afectado a las personas según su condición étnica, y encontrar modos adecuados de generar puentes de perdón y reconciliación, desde 
el conocimiento de sus particularidades. Sobre este tema, Montoya-Ruiz (2008), Patiño, Álvarez y Agudelo (2011), y Díaz-Granados (2015), proponen que las múltiples violaciones a los derechos fundamentales de los niños, niñas y jóvenes que hicieron parte de los grupos armados, se mantienen aún en silencio, y ellos y ellas sienten miedo de dar a conocer sus historias de vida; dicen también que en la actualidad se sigue discutiendo sobre la voluntariedad de algunos de ellos de pertenecer a los grupos armados ilegales.

Por otra parte, respecto al género también existen diferentes afectaciones para hombres y para mujeres, porque sin duda el impacto y las formas de generar procesos de educación para la paz, requieren del conocimiento de las diferencias por todas las variables de afectación diferencial, tales como el género.

Un elemento inesperado surgió a propósito de la cuestión de las consecuencias de la vinculación a un grupo armado ilegal: el $42 \%$ de las niñas entrevistadas expresó que se consideraba una obligación atender sexualmente a superiores en mando. Entre las prácticas que identificaron como el «deber» de atender sexualmente a un superior jerárquico, las niñas describieron el tocamiento, la actividad sexual indeseada, el manoseo, la servidumbre y el tráfico sexual (eran compartidas con otros hombres dentro y fuera de la organización por el intercambio de favores, el acuerdo con socios de negocios, el acceso a bienes o como un mecanismo para acceder a información) (Springer, 2012, p. 48).

Las condiciones que el conflicto trajo a las personas, por ser hombres o mujeres, representan diferencias tanto en su impacto como en las respuestas que debe dar la comunidad educativa, teniendo en cuenta que los sujetos cuentan con huellas particulares que la guerra les ha dejado. Respecto al territorio, se reconoce que las poblaciones pertenecientes a algunos territorios son más vulnerables a verse afectadas por el conflicto armado:

Sumados los factores de vulnerabilidad y riesgo, un niño o una niña indígena tiene 674 veces más posibilidades de verse directamente afectado por el conflicto armado o de ser reclutado y usado por un grupo armado ilegal o una banda criminal que cualquier otro niño en todo el país. (Springer, 2012, p. 20)

Los factores por los cuales fueron secuestrados, reclutados y en general afectados por el conflicto armado son diversos, según los territorios en los que se ubican sus comunidades; es importante develar que las afectaciones por territorio son diferenciales y requieren una atención específica de parte de las comunidades educativas, reconociendo que el país se ha visto principalmente afectado en zonas rurales, y aunque con menor impacto, también en las zonas urbanas.

Todas las personas afectadas por el conflicto armado, principalmente los niños y niñas, son víctimas y no victimarios. Según el informe de Springer (2012), la interacción de niños y niñas con los grupos armados en pocos casos es voluntaria, y aún si es voluntaria, es a causa de fallas estructurales de sus comunidades y del país. Sin embargo, es diferente el impacto que el conflicto armado ha tenido sobre personas reclutadas, que sobre personas desplazadas o que han perdido a sus familias en medio del conflicto; aun así, todos pueden considerarse hoy sobrevivientes de guerra.

Ospina-Ramírez y Ospina-Alvarado (2017) expresan que los niños y niñas han sido principalmente percibidos como sujetos pasivos que solamente reciben las disposiciones de un mundo creado por las personas adultas, pero que es posible desarrollar investigaciones donde sus voces sean visibilizadas, teniendo un mayor impacto en las maneras de transformar las huellas de la guerra en oportunidades de creación conjunta de una nueva escuela, una nueva comunidad y un nuevo país, desde sus voces. Al respecto, Durán-Strauch (2017) expone:

Un gran obstáculo para avanzar en garantizar el derecho a la participación, es la concepción que tienen los sujetos adultos sobre la infancia, incluidos los funcionarios y funcionarias de la administración; persiste una limitación cultural para verlos como sujetos activos y participantes. (Durán-Strauch, 2017, p. 887)

Es claro que los niños, niñas y jóvenes tienen otras maneras de expresar sus ideas y otras formas de interpretar la realidad respecto a las maneras que tenemos los individuos adultos; sin embargo, son voces válidas, importantes y relevantes para la construcción de nuevos mundos y de otras formas de asumir los retos que implican —en este caso- los acuerdos de paz. 


\section{DAVID ARTURo OSPINA-RAMÍREZ - SERGIO LÓPEZ-GONZÁLEZ - \\ SANDRa Bibiana BuRgos-LaItón - José ALEJANDRo MADERA-RUIZ}

\section{La educación como escenario de transición para la construcción de paz}

Las escuelas como estructuras que hacen parte de una macro-estructura social, se convierten en los escenarios de transición donde las personas dejan de lado los caminos de la violencia y los caminos que el mundo les ha enseñado, permitiendo que tengan apertura a nuevas posibilidades de construir la realidad que desean vivir, y de generar transformaciones en sus comunidades más íntimas y cercanas.

Es importante que los escenarios educativos y sus agentes asuman una mirada compleja y crítica del fenómeno del conflicto. Parafraseando a Lykes (2003) en Alvarado et al. (2012), es importante que los agentes educativos recalquen los factores psicológicos de afectación, además de ampliar la mirada y comprender todos los demás aspectos que se ven impactados en la vida de la persona afectada por el conflicto: su cultura, su economía, su familia, sus relaciones, su manera de interpretar y vivir el mundo. Las comunidades educativas deben ampliar la comprensión sistémica y compleja de las afectaciones del conflicto armado de la comunidad estudiantil existente y de las personas vinculadas o afectadas por el conflicto en el territorio regional, abordando el componente psicosocial y profundizando en el conocimiento de la realidad de las personas, principalmente los niños, niñas y jóvenes, y sus comunidades próximas.

En las últimas décadas el concepto de reconciliación ha sido atribuido a procesos de transformación institucional que se desarrollan con el fin de pacificar conflictos armados o proveer los insumos para la ocurrencia de transiciones hacia la democracia. Estos procesos implican la resolución de múltiples problemáticas relacionadas con el enfrentamiento del pasado violento y la consolidación de unas nuevas reglas de juego, por medio de las cuales se establezcan garantías para la convivencia (GarridoRodríguez, 2008, pp. 125-126).

Son las instituciones educativas las que deben realizar transformaciones estructurales que permitan construir o fortalecer los procesos de perdón para la reconciliación. En tal sentido, encontramos que, en este momento, muchos escenarios educativos - incluyendo las escuelas - tienen en sus aulas estudiantes víctimas de la guerra, niños, niñas y jóvenes ex combatientes y también hijos e hijas de personas sobrevivientes de la guerra colombiana. Es por ello que se hace necesaria una mirada desde una educación local, ideada al interior de las comunidades desde el pensamiento latinoamericano, liberador, crítico, reflexivo; donde el educador o educadora, los niños, las niñas y las gentes jóvenes construyan nuevos escenarios de formación desde sus posibilidades, desde sus capacidades y desde sus potencialidades.

Puede decirse que una pedagogía apoyada en una relación de alteridad y en lo dialógico o conversatorio, puede suponer un despliegue de dispositivos pedagógicos que pudieran orientarse hacia la constitución y mediación de una experiencia de sí o subjetivación, sólo que dicha pedagogía tiene un elemento, posiblemente de carácter complementario, la experiencia del otro (Valera-Villegas, 2001, p. 28).

Cabaluz (2014) propone que la Pedagogía pensada para la emergencia del otro y de sus acontecimientos de vida, de sus experiencias, sus expectativas y sus capacidades, permite la generación de otras formas de construir el escenario de lo educativo:

Entonces el respeto al Otro y la nueva generación es un punto de partida para una pedagogía que libera las capacidades que tiene alguien de tener un nuevo proyecto de existencia, y eso es una actitud creativa, que es lo que hay que enseñar filosóficamente y éticamente, luego lo demás son instrumentos empíricos de cómo puedo enseñar mejor. (Cabaluz, 2014, p. 142)

En este sentido, las comunidades educativas tomarían una postura de alteridad, cuando en el dialogismo se generen comprensiones sobre las experiencias que han vivido estas personas, $\mathrm{y}$ dichas comprensiones se transformen en estrategias claras para dar respuestas adecuadas que logren su vinculación y continuidad en el proceso educativo, fortaleciendo sus capacidades para la transformación y haciéndolas visibles, permitiendo que los agentes externos encuentren los fuertes vínculos y puentes que se han construido entre las instituciones educativas y los sujetos afectados por la violencia. 
Bedoya, Builes y Lenis (2009) expresan que la educación permite que cada sujeto emerja desde el interior de sus potencias, narrándose desde las capacidades y reconociendo también que la educación fortalece una narración más centrada en cada individuo, permitiendo que cada quien se narre desde su perspectiva, y también facilitando que desde la otredad cada uno aporte a la construcción de un relato colectivo. Lo anterior lleva a considerar la importancia que tienen los relatos colectivos e individuales respecto a la historia de la guerra vivida en Colombia, las nuevas maneras de asumir los retos actuales para la transformación, y las expectativas de un nuevo país en un contexto posible de construcción de paz.

En este aspecto, es necesario establecer vínculos relacionales más allá de los vínculos institucionales generados entre el niño o niña y su comunidad. Garantizar posibilidades para el fortalecimiento y el despliegue de los niños y niñas va más allá de ofrecer programas de financiación, becas o sustentos financieros que les permitan ingresar al sistema educativo, sin desconocer que este es un gran aspecto a ser tenido en cuenta en los procesos de inclusión educativa, en un contexto de post-conflicto. Pero las comunidades educativas requieren un análisis más profundo y elaborado, un acercamiento a su población estudiantil y a sus contextos, para generar propuestas de desarrollo y vinculación adecuadas a las particularidades de la colectividad, en este caso, comunidades afectadas por la guerra. Además, es importante generar procesos de narración individual y colectiva donde cada quien relate sus experiencias de vida y en ellas reconozca posibilidades para construir paz y para permitir la emergencia de expectativas de una vida distante de la violencia.

Hacer efectivo el acceso a la educación exige garantizar que todos los niños, niñas y jóvenes tengan, en primer lugar, acceso a la educación, pero no a cualquier educación, sino a una educación de calidad con igualdad de oportunidades. Son, justamente, esos tres elementos los que definen la inclusión educativa o educación inclusiva (...). Avanzar hacia la inclusión supone, por tanto, reducir las barreras de distinta índole que impiden o dificultan el acceso, la participación y el aprendizaje, con especial atención en los estudiantes más vulnerables o desfavorecidos, por ser los que están más expuestos a situaciones de exclusión (Homad, 2008, p. 1).

Sin asumir una mirada victimizante o asistencialista, las escuelas y toda su comunidad se convierten en uno de los escenarios de mayor importancia en el desarrollo humano de las personas, más aún en los niños y niñas afectados o vinculados de alguna forma al conflicto armado. Aunque es el Estado el primer escenario que debe ser garante de derechos, no deben esperar las escuelas e instituciones educativas las respuestas del gobierno; por el contrario, deben desde sus posibilidades facilitar procesos que permitan generar una inclusión educativa $\mathrm{y}$, además, favorecer desde allí los procesos de reconciliación y perdón en todos los miembros de la comunidad educativa, por ejemplo, desde la formación y la sensibilización de la Memoria y desde posturas pedagógicas para la paz. Es labor de las instituciones educativas construir puentes que logren restaurar vínculos y resarcir los daños que la guerra ha causado en las vidas de los niños, niñas y jóvenes, permitiendo que ingresen a los contextos educativos; $y$ fortalecer su desarrollo humano desde el reconocimiento de sus experiencias de vida, de sus comprensiones de la realidad vivida y de sus expectativas futuras, para que logren reconciliarse con la sociedad y el mundo que les rodea, asumiendo una mirada crítica, comprensiva, una mirada que desde el perdón facilite la generación de transformaciones en sus vidas y en las vidas de sus comunidades, favoreciendo el despliegue de sus capacidades para convertirse en agentes de cambio.

Vélez y Herrera (2014) proponen que es importante que la educación colombiana establezca una pedagogía para la Memoria, desde el reconocimiento de una historia y partiendo de allí, la escritura de una nueva manera de construir el país desde lo educativo. En un mismo sentido, Herrera y Pertuz (2015) manifiestan que existen fuertes recursos en el campo de lo narrativo, especialmente desde el asumir la capacidad de escuchar de manera diferencial los relatos y las voces de las personas afectadas por la violencia, por ejemplo, dando posibilidades de visibilización a las voces de las mujeres. Lo anterior ofrece expectativas positivas al futuro incierto de la educación en un contexto de posguerra, 
pues se encuentra que en las voces de los niños, niñas y jóvenes existen reconocimientos históricos relacionados con la memoria, pero también futuros posibles desde sus capacidades para transformar su realidad; además, los autores sugieren que no todas las voces hablan de lo mismo, no todas las personas transitaron los mismos caminos de guerra de la misma manera; es por ello que, escuchar desde lo diferencial, es decir, desde el reconocimiento de las afectaciones del conflicto en unos y otros, por su etnia, su territorio, su etapa de desarrollo y su género, permite que los agentes educativos construyan con cada estudiante diversas maneras de aportar a la paz y transformar las huellas de la guerra de modo específico, y de esta forma beneficiar también la transformación colectiva de las experiencias de guerra.

Para las escuelas rurales y para las escuelas urbanas, la educación en un contexto de posconflicto implica esfuerzos centrados en la formación crítica y reflexiva, en la construcción de pedagogías para la memoria, en la sensibilización de las comunidades de acogida de los individuos sobrevivientes de guerra para la acción sin daño y para no caer en la revictimización. Sin embargo, existen algunos esfuerzos particulares que ambas deben realizar; por ejemplo, en el caso de las escuelas rurales, sus comunidades han conocido de cerca las huellas de la violencia, de manera que su trabajo estará más orientado a la reconciliación, al perdón y a la no repetición por medio de procesos formativos en convivencia y construcción de paz; mientras que para las escuelas urbanas el reto está en la sensibilización de la comunidad que no ha conocido de manera directa el impacto de la guerra, la educación histórica y el fortalecimiento de la solidaridad, la compasión y la ética del cuidado del otro, propuesta por Alvarado y Ospina (2014).

\section{El potencial creativo de la niñez para la transformación}

Ospina-Ramírez y Ospina-Alvarado (2017), manifiestan que los niños y niñas cuentan con potenciales y capacidades para construir paz, aun cuando el contexto en el que se encuentran ha dejado marcas y huellas de violencia. En esta investigación, los niños y niñas que participaron del Patía (Cauca), tienen mayor relación y afectación con la violencia que generó el conflicto armado; en el caso de los que participaron en la ciudad de Manizales, no han tenido ninguna afectación por la violencia de manera directa en términos del conflicto armado. En este sentido, Alvarado et al. (2012) plantean la forma en que los niños y niñas de la zona rural generan procesos de construcción de paz, a partir del reconocimiento de sus recursos y potencialidades individuales y grupales; en el caso de la investigación, el dibujo es una forma en la que los niños y las niñas de los diferentes territorios intervenidos muestran su realidad; estas potencialidades permiten generar relaciones en las que ellos y ellas participan activamente en la creación de sus propios recursos, individuales y grupales. De manera que los niños y niñas, en su interacción social, pueden participar con sus familias y agentes educativos en procesos de cambio para la construcción de paz.

Los dibujos, desde las representaciones simbólicas de los niños y niñas, son herramientas necesarias para comunicar y educar, desde sus voces, a las generaciones presentes y futuras, sobre los hechos y las experiencias vividas, además de sus expectativas y sus esperanzas de un país que viva relaciones pacíficas. El juego y las artes son el lenguaje propio de la infancia, pues estos son medios espontáneos de expresión, representación y comunicación de su mundo; por medio de estas herramientas se evidencia un profundo interés de los niños y las niñas, en convertirse en partícipes de un futuro diferente al pasado que han vivido. Es importante resaltar los aportes que realizan OspinaAlvarado, Carmona-Parra y Alvarado-Salgado (2014), que permite comprender la importancia de las expectativas de paz que los niños y niñas plantean en sus dibujos:

Aparece la solidaridad, la inconformidad, la resistencia frente a lo vivido, el interés por participar en un futuro distinto, la importancia dada a la acción colectiva, las redes protectoras, el cuidado por la naturaleza y el interés de niños por ser nombrados desde sus potencias y no desde sus carencias principalmente en la zona rural, estos elementos aportan a la construcción de paz y que también están presentes entre niños y niñas que habitan el contexto del conflicto armado y entre sus relaciones significativas. (Ospina-Alvarado, Carmona-Parra, \& Alvarado-Salgado, 2014, p. 57) 
Para lageneración de espacios de creatividad, es necesario crear escenarios donde el reconocimiento de la violencia — desde una mirada apreciativa - permita identificar también prácticas de paz que han facilitado, por ejemplo en el caso colombiano, la dejación de armas por parte de los grupos armados. Para Alvarado, Ospina-Alvarado y Sánchez-León (2016), comprender la realidad es transformarla; desde esta perspectiva se considera entonces que la indagación por los imaginarios de los niños y niñas sobre el posconflicto y la construcción de paz, necesariamente genera también transformaciones en dichos imaginarios y en su postura como agentes de cambio.

El reconocimiento y comprensión de los contenidos y formas mediante las cuales los niños y niñas que viven en contextos de conflicto armado construyen y narran de manera generativa sus identidades y subjetividades, de modo tal que logran movilizar procesos de paz, reconciliación y democracia, el interés práxico del estudio integró el ejercicio de comprender las formas y sentidos particulares de construcción de la identidad y la subjetividad, que hacen visibles y audibles elementos de la realidad que no han sido nombrados: aquellas prácticas singulares, aquellos modos de ser en el mundo que han logrado instituir procesos de paz, reconciliación y democracia en medio de un contexto de guerra (Alvarado et al., 2016, p. 997).

Las creaciones propias a partir de las vivencias son tan fuertes que son capaces de movilizar cualquier iniciativa de cambio, por lo cruel y triste que puede ser la realidad de cada quien, especialmente de los individuos directamente afectados por el conflicto armado. Es de resaltar que, a pesar de vivir en medio de la guerra, los niños y niñas revelan de forma espontánea sentimientos de reconciliación, de unión y de amor; como consecuencia del proceso de sanación particular y sobre todo dando a entender a través de sus construcciones cómo es posible cambiar ese contexto de guerra. La postura de los sujetos adultos respecto a las vivencias de la niñez en un país que ha experimentado la violencia en todo su territorio, debe considerar que:

La infancia y la adolescencia son etapas cuyas tareas de desarrollo demandan de contextos sociales protegidos, que estimulen el despliegue de potencialidades cognitivas, físicas, emocionales, sociales y éticas. Son edades en las que el tiempo perdido y las afectaciones en el desarrollo tienen implicaciones significativas y perdurables. (Cifuentes, 2015, p. 3)

La protección para la niñez y la juventud en el posconflicto es un factor fundamental en lo relacionado con la garantía de no repetición; sin embargo, la firma de los Acuerdos de Paz en sí misma y el proceso del posconflicto, no garantizan que los niños y las niñas no vuelvan a vivir escenarios de violencia, a pesar de las garantías que ofrece el Acuerdo sobre la no repetición. Esto permite comprender que la sociedad puede, desde sus capacidades instaladas, desde sus fortalezas y recursos, garantizar que ellos y ellas puedan desplegar sus potencialidades y mitigar el impacto de las afectaciones de la violencia. Allí también la creatividad de las personas adultas, de la comunidad, de los agentes educativos y familias, juega un rol trascendental, considerando que muchas comunidades no cuentan con las condiciones políticas, económicas y sociales adecuadas, lo que implica un mayor reto para la transformación desde la mirada crítica de estos agentes. Son entonces el trabajo colectivo, participativo y creativo de toda la comunidad educativa, factores que aportan a la transformación de la guerra desde el cuidado de los niños, niñas y jóvenes, para la potenciación de sus capacidades y la generación de oportunidades de vida diferentes a las planteadas por la violencia.

Respecto a la creatividad desde la expresión narrativa y artística de los niños y niñas, Naranjo (2015) expresa que es importante:

Activar el intelecto y espolear la imaginación para que el alumno encuentre sus propias respuestas, no las que supone que esperamos de él, esas «políticamente correctas» que a todos nos dejan «satisfechos». En los encuentros me esforcé contra mis naturales limitaciones (y deformaciones) a preguntar desde esa zona más riesgosa y fértil para nutrir la compañía que como en una fiesta nos dábamos. (Naranjo, 2015, p. 10)

Desde esta mirada, quienes acompañan el acto educativo o social asumen una mirada comprensiva y apreciativa de las expresiones de los niños, sin esperar una respuesta específica y determinada sobre el campo; por el contrario, presentando apertura a las propuestas, imaginarios, prácticas y sentidos de 


\section{DAVID ARTURo OSPINA-RAMÍREZ - SERGIO LÓPEZ-GONZÁLEZ - SANDRA BIBIANA BURGOS-LAITÓN - José ALEJANDRO MADERA-RUIZ}

paz que los niños y niñas manifiestan. Ellos y ellas hablan desde sus saberes, desde sus experiencias de vida y desde lo que ven. En este sentido, los niños y niñas reconocen una realidad que constantemente los aqueja, pero también ven otro panorama diferente al que han vivido; la paz es para ellos y ellas una forma de mejorar su calidad de vida.

Enseñar sobre la paz permite desarrollar competencias en los alumnos que les sirvan para poder convivir en cualquier tipo de sociedad. Por esto se deben fomentar las competencias generales como la comunicación oral, la lectura y la escritura, las competencias de integración social, las actitudinales, las aptitudinales y las lúdicas. En cuanto a las competencias específicas se les deben desarrollar las cognitivas, las emocionales, interpretativas, las analíticas, las argumentativas, las propositivas y las ciudadanas (Salamanca, Rodríguez, Cruz, Ovalle, Pulido, \& Molano, 2016, p. 7).

Los niños y niñas que están expuestos a la violencia cotidianamente en sus barrios o comunidades, pueden desplegar su creatividad desde sus propias miradas y desde sus acciones propositivas para el cambio. Educarlos para la paz permite generar procesos de empatía, entendida esta como la capacidad para sentir algo similar a lo que sienten los otros, y como la posibilidad de reconocer al otro como diferente; este proceso es fundamental para la convivencia y la creatividad participativa, donde las ideas de todos y todas tienen cabida.

Para el despliegue de las capacidades creativas de los niños y niñas es clave el rol que asuma el agente educativo; en palabras de Grandas, Parra, Pineda y Romero (2016):

Los agentes educativos tienen un rol fundamental en el reconocimiento y la inclusión de los niños o niñas afectados por el conflicto armado en la medida en que logran establecer vínculos de acercamiento y confianza que aportan a la integración, aceptación y reconocimiento de los y las estudiantes al manifestar signos de confianza, ternura, compasión o empatía y al mostrar interés y deseo por conocer los sentimientos, las emociones y las situaciones que expresan los niños y las niñas en el contexto escolar. Es ahí cuando surgen habilidades individuales, relacionales, comunicativas y performativas que permiten ir superando las dificultades y transformando los conflictos en situaciones de paz. (p. 41)

Entonces la creatividad, como recurso, requiere también de unos contextos que motiven su despliegue donde las voces de los niños y niñas tengan sentido desde la comprensión empática por parte del individuo adulto. La creatividad como recurso compartido facilita la construcción de paz desde las diversas visiones de niños, agentes educativos, familias y otros actores.

\section{Ruta metodológica}

La investigación fue de tipo cualitativo; asumimos una postura hermenéutica comprensiva, tomando como referencia el estudio de caso para su desarrollo. En el proyecto prestamos atención a las posturas que los niños y niñas tienen sobre la construcción de paz en el contexto del posacuerdo firmado por el Gobierno colombiano y uno de los más grandes grupos armados, las Farc-EP. Para Saavedra (2005), la investigación hermenéutica es una manera de comprender una realidad dada, encontrando recursos para conocer y develar aspectos desde el lenguaje, desde las maneras en que se relata esa realidad, además de ser una investigación que permite permear contextos humanos para interpretar las maneras en que se mantienen vivos por medio de las relaciones. Desde lo narrativo, Biglia y Bonet-Martí (2009) proponen que una misma historia puede ser narrada desde múltiples voces y miradas, por lo que la narrativa cobra importancia a la hora de comprender una realidad social.

Desarrollamos la investigación en tres fases y conformamos un equipo de trabajo de doce investigadoras e investigadores. La primera fase del trabajo consistió en un levantamiento del Estado del Arte, por medio de la revisión documental de investigaciones nacionales realizadas en el campo. También en esta fase construimos los instrumentos de investigación, que consistieron en tres talleres creativos y una entrevista semiestructurada, y que validamos desde un pilotaje realizado en la ciudad de Manizales. El primero de estos talleres consistió en indagar por lo que los niños 
y niñas comprenden, conocen o han escuchado sobre el conflicto armado, por medio de dibujos, relatos y ejercicios colectivos. En el segundo taller indagamos por lo que los niños y niñas conocen de los Acuerdos de Paz y su postura; de igual forma, empleando el dibujo, la pintura y los relatos. Finalmente, el tercer taller consistió en la indagación de lo que los niños y niñas están dispuestos a aportar para la construcción de paz y la transformación de una cultura de guerra. En una segunda fase aplicamos dichos instrumentos en una población urbana en la ciudad de Manizales, en una población urbana en la ciudad de Popayán y en una zona rural del Patía, Cauca. La última fase consistió en el análisis de los escritos, narrativas y dibujos de los niños y niñas participantes (entre 7 y 12 años de edad), por medio de un análisis categorial de la información, siguiendo el orden de cada uno de los talleres y empleando el Atlas Ti para su debido procesamiento, teniendo en cuenta que los dibujos de los niños y niñas estaban acompañados de pequeñas descripciones realizadas por ellos mismos. Al finalizar dicho análisis, devolvimos a la comunidad la información por medio de una Galería de Arte por la Paz, expuesta en el Centro Colombo Americano de Manizales.

\section{Principales hallazgos y resultados}

Un aspecto importante a resaltar en esta investigación, fue el trabajo que adelantamos en zonas rurales y en zonas urbanas, comprendiendo que el impacto que ha traído la violencia es radicalmente diferencial; por ejemplo, entre Caldas y Cauca, e incluso en el mismo departamento, los impactos de la guerra en niños y niñas que han estado en el campo, son diferentes a los que han vivido quienes habitan en las ciudades.

\section{Imaginarios de la paz en el territorio}

Las condiciones en que viven los niños y niñas en la actualidad, determinan la manera en que interpretan una transformación en el país, llevando esta transformación a un cambio más próximo a sus comunidades y territorios. Un factor importante que encontramos en varias representaciones gráficas de los niños, es que imaginan la paz como una transformación del territorio que habitan:

Imagen 1. Dibujo de niña de 11 años, Manizales zona urbana

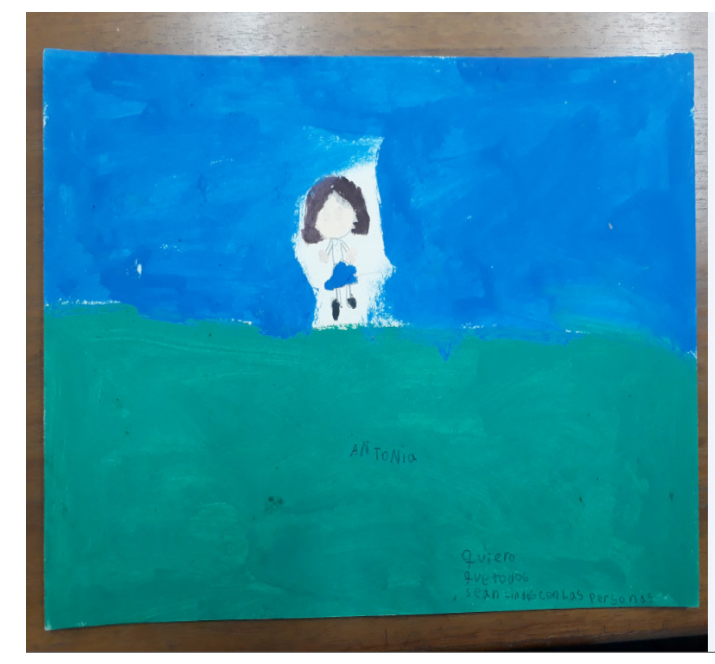


Imagen 2. Dibujo de niño de 8 años, Patía zona rural

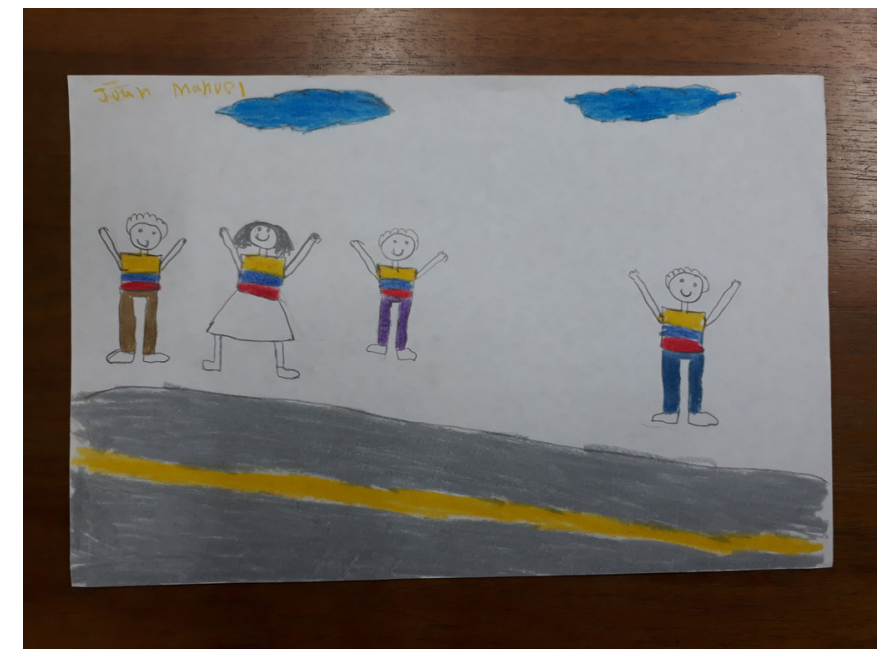

Como se refleja en los anteriores dibujos y en diferentes representaciones de los niños y niñas, quienes se encuentran en zonas rurales imaginan la paz en las ciudades, representando calles, edificios, casas en zonas urbanas, etc. Mientras que quienes viven en zonas urbanas, como el caso de Manizales, imaginan la paz en el campo, representando bosques, playas, lugares próximos a la naturaleza. Se podría interpretar que la transformación que los niños y niñas esperan del país en un contexto de posconflicto, se relaciona con lo que ellos esperan que mejore en sus territorios; en el caso de los participantes de zonas rurales esperan desarrollo, vías y mayor cercanía a los imaginarios urbanos; los niños y niñas que participaron en sectores urbanos, imaginan un país con mayor naturaleza y con otros escenarios y paisajes más cercanos a lo que viven los sectores rurales.

\section{La paz asociada a escenarios tranquilos y armónicos}

En uno de los talleres pedimos a los niños y niñas que en sus dibujos escribieran lo que esperaban del país después de la firma de los acuerdos de paz. Tanto en zonas urbanas como rurales, el cuidado ambiental fue un factor que ellos y ellas asociaron a la construcción de paz y a la transformación de la cultura.

Imagen 3. Dibujo de niño de 10 años, Manizales zona urbana

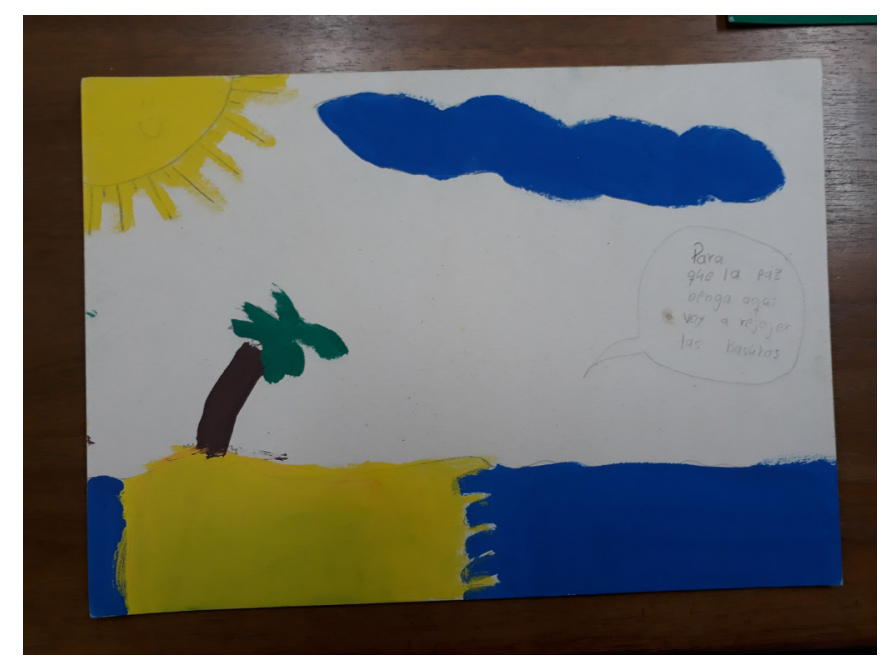


En diferentes dibujos, los niños plantean que la paz para ellos significa armonía y tranquilidad, asociándola con lugares que les generan esas sensaciones, y planteando que el cuidado de esos escenarios es lo que garantiza la construcción de paz. De igual forma, la armonía y la tranquilidad la asocian con la construcción de paz desde las emociones que esto les suscita:

Imagen 4. Dibujo de niño de 7 años, Popayán zona urbana

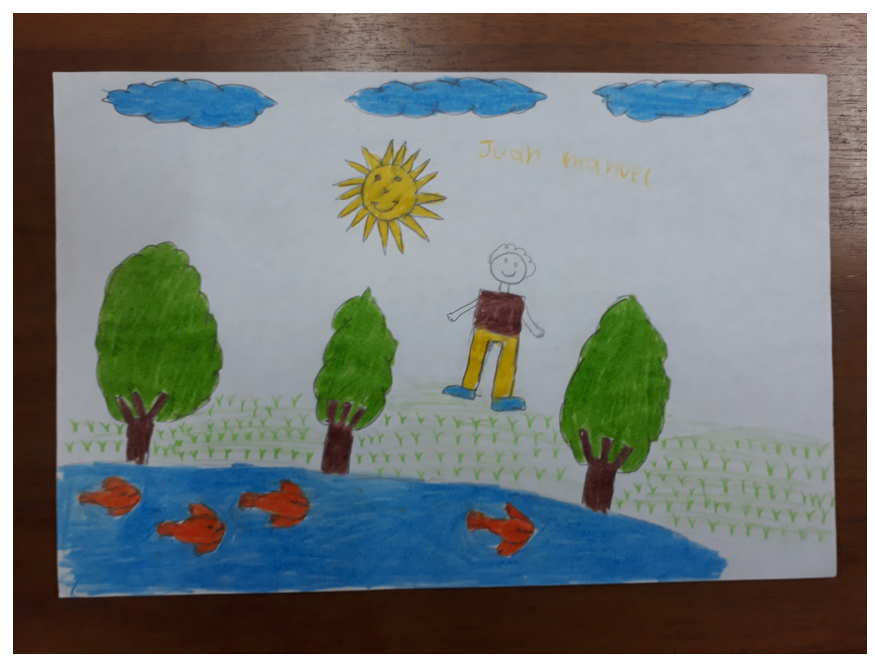

Detallamos a continuación el relato que acompaña este dibujo:

Este lugar me genera paz, porque no se escuchan ruidos ni estruendos, se escucha el canto de las aves, el ruido del río, esto me mantiene tranquilo y fresco, estar bajo la sombra de un árbol. Para que en este lugar exista paz, yo debo cuidarlo, ojalá no corten árboles, así hay armonía y no hay guerra (Relato de niño de 7 años, Popayán zona urbana).

Como se evidencia en el relato, la tranquilidad es una de las principales características que los niños y niñas relacionan con la paz y con los lugares que les generan este tipo de sensaciones. También los lugares que encontraron en sus imaginarios de paz, se relacionan con la comunidad que atraviesa y que habita dichos escenarios.

\section{La construcción de paz en la comunidad que habitan los niños y las niñas}

Otros escenarios que encontramos en los imaginarios de paz en un contexto de posconflicto, son los más cercanos a la comunidad en la que se desenvuelven y se desarrollan los niños y niñas: la escuela, el barrio y el hogar; esto permite anotar un hallazgo importante, y es que los niños y niñas perciben la paz en sus contextos relacionales próximos, de manera que la estructura en que fueron desarrollados los Acuerdos de Paz, cuenta con un factor social y cultural clave: la infancia desea construir paz desde sus comunidades, como se evidencia a continuación: 
Imagen 5. Dibujo de niña de 11 años, Patía zona rural

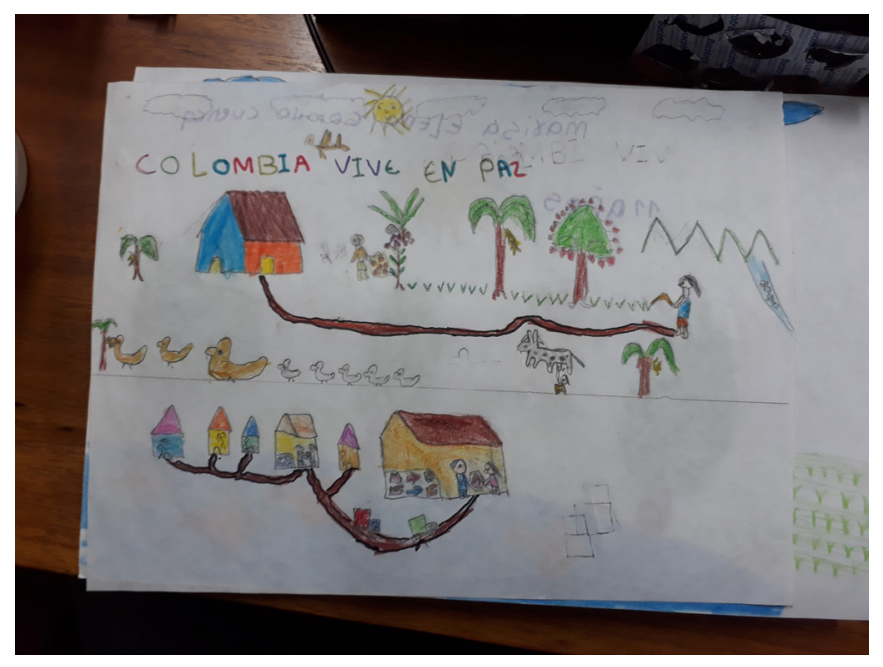

En el dibujo se identifica cómo la niña imagina la paz desde su hogar, desde su contexto, desde las relaciones con sus vecinos y desde su relación con la naturaleza. La relación con la comunidad se ejemplifica en diversas representaciones que realizaron los niños:

Imagen 6. Dibujo de niño de 10 años, Popayán zona urbana

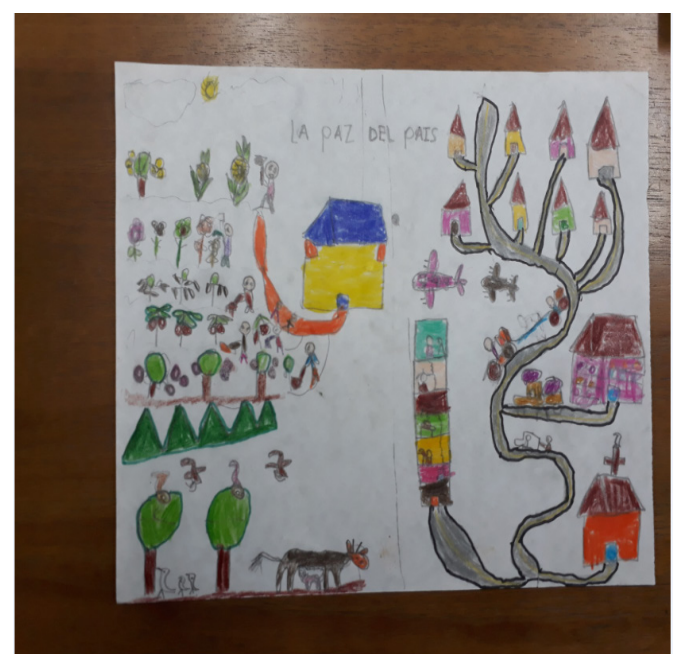

La relación que dibuja el niño en esta representación, evidencia el desarrollo como un factor clave para sectores urbanos y rurales del país; de igual forma, la comunidad se refleja en sus diferentes actividades cotidianas, interpretando que el niño imagina una paz cercana a la vida cotidiana de los colombianos, donde todos tienen cabida. 
Imagen 7. Dibujo de niño de 10 años, Popayán zona urbana

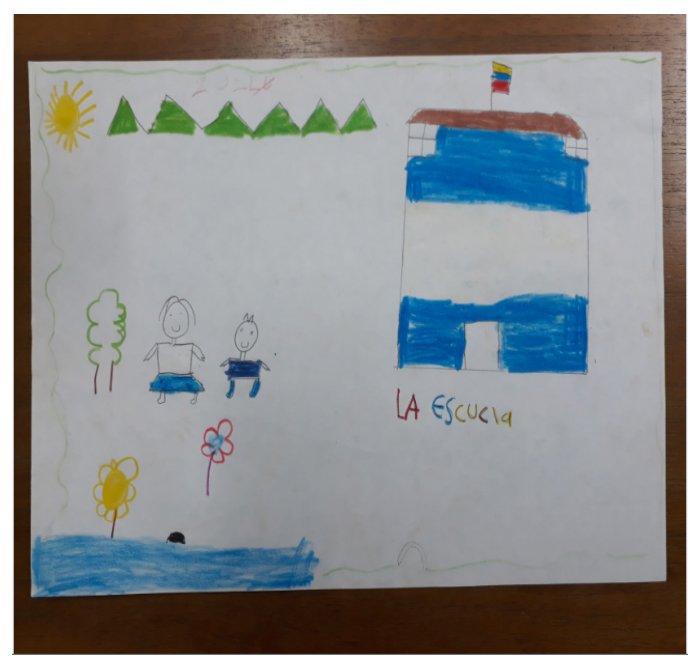

La escuela se convierte en uno de los escenarios principales donde los niños y niñas se imaginan la paz, elaborando diversos relatos relacionados con el cuidado del otro, con el afecto y con la tolerancia a las diferencias. Los niños y niñas en esta investigación manifestaron también posturas claras respecto a los Acuerdos de Paz y sus aportes para la transformación de la naturalización de la violencia que se ha generado y arraigado a la cultura del país.

\section{Posturas de los niños y niñas respecto a los acuerdos de paz}

Los niños y niñas que participaron en el proyecto, según su nivel de afectación por el conflicto, manifestaron acuerdo o desacuerdo respecto al proceso de paz. Los de la ciudad de Manizales mostraron más escepticismo y temor respecto al proceso de paz; sin embargo, en sus escritos expresaron estar dispuestos a aportar para la transformación del país con la firma de los acuerdos.

"Yo me imagino caminando por Colombia más tranquilo y viajando a donde yo quiera. Debemos cuidar nuestra humanidad, pero también la naturaleza. Estamos destruyendo nuestro nevado, los frailejones... y evitar las quemas. Me comprometo a respetar a todos los desmovilizados que conozca, pero más importante voy a cuidar la naturaleza, los nevados, los animales, las plantas y los seres vivos.» (Escrito de niño de 12 años, Manizales zona urbana)

"Yo me imagino que después de los acuerdos de paz, Colombia sería menos peligrosa, la gente tendría menos miedo y más confianza en la gente que nos rodea. Yo me comprometo, ayudando a los niños y a la gente de la guerrilla cuando vuelvan a mi colegio o a mi sector de vivienda, los apoyaré para que se adapten al ambiente y tengan nuevos amigos. Gracias por leer, Colombia.» (Escrito de niño de 11 años, Manizales zona urbana)

En el caso de Popayán y de la zona rural de Patía, los niños y niñas expresaron en sus escritos un respaldo a los Acuerdos de Paz, y manifestaron su conformidad con la finalización del conflicto de manera armada.

Señor Juan Manuel Santos Calderón

Lo saludo y espero que se encuentre muy bien de salud y en compañía de su familia. La presente es para informarle que, sí estoy de acuerdo con los diálogos de paz, porque en nuestro país no habrá más derramamiento de sangre, no más secuestros, no más violencia. (Relato niño de 10 años, Popayán zona urbana)

Teniendo en cuenta que en esta actividad les pedimos a los niños y niñas que escribieran cartas al presidente — quien lideró el proceso de paz-, desde lo que creían que iba a ser el acuerdo y cómo iba a afectar a sus comunidades, y que los niños y niñas del Patía (Cauca) mostraron interés en la 
firma de los acuerdos, concluimos que ello se corresponde con el nivel de afectación que ha tenido el Cauca por el conflicto armado:

Presidente de la república de Colombia, Cordial saludo.

Le pido a usted, que por favor no ordene que continúe la guerra, ni derramamiento de sangre. Nosotros los niños le pedimos la paz por Colombia, por los estudiantes del campo, barrio y ciudades. Le pedimos que siga firme en el proceso de paz. Muchas gracias, ojalá que nos escuche. (Relato niña de 10 años, Patía zona rural)

En los tres nodos o grupos de trabajo, los niños y niñas manifestaron disposición para recibir a otros niños y niñas víctimas de la violencia o que hubiesen hecho parte de los grupos armados. También identificamos que, aunque los niños y niñas que se encuentran en la ciudad tienen más temores que los que se encuentran afectados de manera directa por la guerra, aquellos tienen apertura al cambio y a aportar para la construcción de paz en el país y en sus territorios.

\section{Conclusiones y comentarios finales}

La investigación nos permitió encontrar que los niños y niñas que se ubican en territorios rurales, expresan en sus dibujos y relatos expectativas sobre los acuerdos de paz en relación con la no repetición de la guerra, y también proyectan en su imaginación que sus territorios tengan mayores facilidades para acceder a la escuela, representando carreteras en sus dibujos, implicando un fuerte trabajo en el sector rural, vías de acceso para sus territorios, facilidades para la movilidad a sus escuelas, mayor seguridad en el desplazamiento. Todo lo anterior, desde nuestra mirada adulta, podríamos denominarlo desarrollo, que se evidencia en los acuerdos de paz respecto al fortalecimiento del sector rural; los niños y niñas lo expresan en términos de tranquilidad, seguridad y posibilidad de llegar más fácil a su escuela. Los niños y las niñas del campo desean permanecer en sus territorios y no desplazarse a territorios urbanos, expectantes de las transformaciones que implican los acuerdos de paz, garantizando que los grupos armados no retornen a sus territorios. Un hallazgo importante en el caso rural es que los niños y niñas no tienen un conocimiento sobre las dimensiones de los Acuerdos de Paz; sin embargo, conocen que estos implican la finalización del conflicto armado.

En el caso de los niños y niñas habitantes de contextos urbanos, evidenciamos un mayor conocimiento respecto a los acuerdos de paz, y a su vez cierta predisposición a la llegada de personas desmovilizadas de los grupos armados a sus territorios, así como algo de inseguridad y miedo a que las personas que pertenecieron a los grupos armados lleguen a causar daños a su comunidad y a sus familias. En el caso urbano encontramos en algunos relatos que el conocimiento que se tiene de los acuerdos de paz ha sido permeado por el acceso a redes sociales y por las posturas que tienen sus familias y docentes. En algunos relatos expresan inconformidad con los acuerdos de paz, pensando que las personas desmovilizadas van a tener oportunidades y mejores condiciones de vida que la sociedad civil que no ha participado de la guerra, lo que evidencia un discurso de las personas adultas que se ha replicado en las concepciones que tienen los niños y niñas.

La investigación permite reconocer que, tanto en escenarios rurales como urbanos, la mirada y la postura de los sujetos adultos respecto a los acuerdos de paz, se asume por parte de los niños y las niñas, quienes en sus relatos expresan sus miedos, expectativas y posturas desde lo que han escuchado de la gente adulta. En el caso urbano, las redes sociales tienen un alto impacto en el discurso de los niños y las niñas respecto a la finalización del conflicto armado. En ambos casos hay desconocimiento de todas las dimensiones de los acuerdos de paz; esto evidencia la importancia de una formación para la paz desde el conocimiento de estas dimensiones, como las garantías en la educación, las posibilidades del desarrollo rural, y las garantías de no repetición del conflicto armado; ello implica un esfuerzo por parte del Gobierno y de las comunidades educativas para brindar información más clara y comprensible, en un lenguaje cercano a los niños y niñas, que les permita asumir una mirada crítica y reflexiva sobre la violencia, la firma de los acuerdos de paz y el post-acuerdo en el país. 
Es importante que los profesores y profesoras, familiares y personas formadoras de niños y niñas, brinden la información de una manera imparcial y que posibilite que ellos y ellas asuman una postura propia sobre los acuerdos de paz y, sobre todo, que les permita reconocer una realidad en la cual los niños y niñas están inmersos y, por ende, como sujetos activos pueden ellos mismos aportar a la construcción de paz desde acciones cotidianas que transformen las dinámicas sociales desde temprana edad.

Para futuras investigaciones en el campo, es importante encontrar un lenguaje claro y de fácil comprensión para los niños y niñas, y de igual forma interpretar sus posturas desde las formas como se expresan por medio de expresiones como dibujos y relatos. También es importante en un primer momento, indagar qué entienden los niños y niñas sobre la paz y la violencia en sus territorios y cómo es la diferenciación entre lo que imaginan, lo que han escuchado y lo que han vivenciado, para poder distinguir sus voces propias y no solamente relatos que vienen de los individuos adultos y que se imprimen en sus voces.

\section{Lista de referencias}

Alvarado, S. A., Ospina-Alvarado, M. C., \& Sánchez-León, M. C. (2016). Hermenéutica einvestigación social: narrativas generativas de paz, democracia y reconciliación. Revista Latinoamericana de Ciencias Sociales, Niñez y Juventud, 14(2), 987-999. doi: 10.11600/1692715x.14207170215

Alvarado, S. V., \& Ospina, H. F. (2014). Socialización y configuración de subjetividades: construcción social de niños y niñas y jóvenes. Bogotá, D.C.: Siglo del Hombre Editores, Universidad de Manizales, Cinde.

Alvarado, S. V., Ospina H. F., Quintero M., Luna M. T., Ospina-Alvarado, M. C. \& Patiño, J. A. (2012). Las escuelas como territorios de paz. Manizales: Cinde.

Bedoya, M. H., Builes, M. V., \& Lenis, J. F. (2009). La acción educativa como acción narrativa. Revista Latinoamericana de Ciencias Sociales, Niñez y Juventud, 7(2), 1255-1271.

Biglia, B., \& Bonet-Martí, J. (2009). La construcción de narrativas como método de investigación psico-social: prácticas de escritura compartida. Forum Qualitative Sozialforschung/Forum: Qualitative Social Research, 10(1), 1-25.

Cabaluz, F. (2014). Filosofía de la liberación y pedagogías críticas latinoamericanas. Entrevista a Enrique Dussel. Cuaderno de Pensamiento Latinoamericano, 21, 136-148. doi: 10.5294/ edu.2016.19.1.4

Cifuentes, M. R. (2015). Niñez y juventud, víctimas del conflicto armado: retos para el trabajo social. Tendencias \& Retos, 20(1), 161-177.

Díaz-Granados, E. T. A. (2015). Traumas asociados a violencia en excombatientes de grupos armados ilegales. Revista de Psicología Universidad de Antioquia, 7(1), 21-34.

Durán-Strauch, E. (2017). Derechos de niños y niñas: del discurso a la política local. Revista Latinoamericana de Ciencias Sociales, Niñez y Juventud, 15(2), 879-891. doi:10.11600/169271 5x.1520623062016. Recuperado de:

http://revistaumanizales.cinde.org.co/index.php/Revista-Latinoamericana/article/view/2465.

Garrido-Rodríguez, E. (2008). El perdón en procesos de reconciliación: el mecanismo micropolítico del aprendizaje para la convivencia. Papel Politico, 13(1). 123-167.

Grandas, A., Parra, O. S., Pineda, M., \& Romero, E. G. (2016). Construcción de paz, democracia y reconciliación con niños y niñas en contextos de conflicto armado. (Tesis de Maestría). Universidad Pedagógica Nacional-Cinde, Bogotá, D. C., Colombia.

Herrera, M. C., \& Pertuz, C. (2015). Narrativas femeninas del conflicto armado y la violencia política en Colombia: contar para rehacerse. Revista de Estudios Sociales, 53, 164-173. doi: http://dx.doi. org/10.7440/res53.2015.12

Homad, G. E. (2008). Inclusión educativa. Revista Electrónica Iberoamericana sobre Calidad, Eficiencia y Cambio en Educación, 12(2), 1-8. 
Montoya-Ruiz, A. M. (2008). Niños y jóvenes en la guerra en Colombia: aproximación a su reclutamiento y vinculación. Revista Opinión Jurídica, 7(13), 37-51.

Naranjo,J.(2015). Los niños piensan la paz. Bogotá, D. C.: Banco dela República. Recuperado de: http:// proyectos.banrepcultural.org/proyecto-paz/sites/default/files/documentos/losninospiensan_la_ paz_web.pdf.

Ospina-Ālvarado, M. C., Carmona-Parra, J. A., \& Alvarado-Salgado, S. V. (2014). Children in context of armed conflict: Peace generating narratives. Revista Infancias Imágenes, 13(11), 52-60.

Ospina-Ramírez, D. A., \& Ospina-Alvarado, M. C. (2017). Futuros posibles, el potencial creativo de niñas y niños para la construcción de paz. Revista Latinoamericana de Ciencias Sociales, Niñez y Juventud, 15(1), 175-192. doi: 10.11600/1692715x.1511016022016

Patiño, M. R. C., Álvarez, N. A., \& Agudelo, N. V. L. (2011). Niñas, niños y jóvenes excombatientes: revisión de tema. Eleuthera, 5, 93-125.

Saavedra, T. R. (2005). La hermenéutica reflexiva en la investigación educacional. Revista Enfoques Educacionales, 7(1), 51-66.

Salamanca, M., Rodríguez, M., Cruz, J. D., Ovalle, R., Pulido, M. A., \& Molano, A. (2016). Guía para la implementación de la cátedra de paz. Bogotá, D. C.: Editorial Santillana, Universidad Javeriana. Recuperado de: http://santillanaplus.com.co/pdf/cartilla-catedra-de-paz.pdf.

Springer, N. (2012). Como corderos entre lobos: que dejen de cazar a los niños y a las niñas. Bogotá, D. C.: Springer Consulting Group.

Unidad para las Víctimas. (2017). Registro Único de Víctimas (RUV). Bogotá, D. C.: Gobierno de Colombia. Recuperado de: http://rni.unidadvictimas.gov.co/?q=node/107.

Valera-Villegas, G. (2001). Escuela, alteridad y experiencia de sí: producción pedagógica del sujeto. Educere, 5(13), 25-30.

Vélez, G., \& Herrera, M. C. (2014). Formación política en el tiempo presente: ecologías violentas y pedagogía de la memoria. Nómadas, 41, 149-165. 